\title{
Protein corona formation around biocatalytic nanomotors unveiled by STORM
}

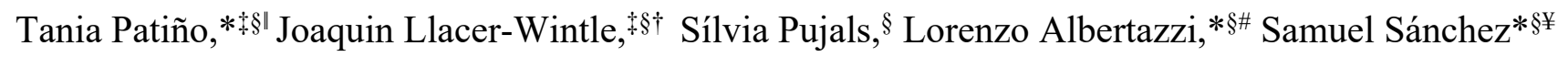 \\ " University of Rome Tor Vergata, Via della Ricerca Scientifica, oo133 Rome, Italy \\ $\S$ Institute for Bioengineering of Catalonia (IBEC), The Barcelona Institute of Science and Technology (BIST), Baldiri \\ i Reixac 10-12, o8028 Barcelona, Spain \\ \# Department of Biomedical Engineering, Institute for Complex Molecular Systems (ICMS), Eindhoven University of \\ Technology, 5612AZ Eindhoven, The Netherlands \\ ${ }^{*}$ Institució Catalana de Recerca i Estudis Avançats (ICREA), Pg. Lluís Companys 23, o8010 Barcelona, Spain
}

Supporting information for this article is given via a link at the end of the document

\begin{abstract}
The interaction of nanoparticles with biological media is a topic of general interest for drug delivery systems and among those for active nanoparticles, also called nanomotors. Herein, we report the use of super resolution microscopy, in particular stochastic optical reconstruction microscopy (STORM), to characterize the formation of protein corona around active enzyme-powered nanomotors. First, we characterize the distribution and number of enzymes on nano-sized particles and characterized their motion capabilities. Then, we incubated the nanomotors with fluorescently labelled serum proteins. Interestingly, we observed a significant decrease of protein corona formation ( $20 \%$ ) and different composition, which was studied by a proteomic analysis. Moreover, motion was not hindered, as nanomotors displayed an enhanced diffusion regardless of protein corona. Elucidating how active particles interact with biological media and maintain their self-propulsion after protein corona formation will pave the way of the use these systems in complex biological fluids in biomedicine.
\end{abstract}

Inspired by nature, catalytic nanomotors that convert chemical energy into motion have been developed in the last decade. ${ }^{1}$ Particularly, enzymes are highly efficient natural catalysts that constitute a very promising strategy to build biocompatible micro- and nanoswimmers that self-propel using bioavailable fuels such as glucose, ${ }^{2}$ $\mathrm{H}_{2} \mathrm{O}_{2}{ }^{3}$ or urea ${ }^{4,5}$ without the need from external power sources. This has opened new possibilities in the use of nanomotors for biomedical applications, and several milestones have been already reached, including enhanced anti-cancer drug delivery, ${ }^{6}$ improved cell uptake, ${ }^{7}$ sensing $^{8}$ and medical imaging. Despite these exciting outcomes, not all enzymes can generate active motion in a highly efficient manner, being urease one of the most powerful enzymes to promote self-propulsion for micro- and nanoparticles. ${ }^{9}$ Urease-powered nanomotors have already been used to enhance anti-cancer drug delivery, ${ }^{6}$ and promote a more efficient cell uptake ${ }^{10}$ targeting and penetration of $3 \mathrm{D}$ spheroids ${ }^{7}$. Additionally, urease-nanomotors are capable of self-propel within biological fluids such as urine ${ }^{7}$ and blood. ${ }^{10}$

Despite these exciting outcomes, some concerns have been raised for their use in biological media and potential biomedical application. ${ }^{11}$ To tackle this issue, the challenges that all nanoparticle delivery systems face when they enter the organism should be considered. Upon nanoparticle administration, proteins, lipids and small metabolites present within the biological fluids instantly adsorb to the nanoparticle surface, forming a protein corona that confers a new biological identity to the nanoparticle, ${ }^{12,13}$ altering their targeting capabilities, ${ }^{14,15}$ cellular uptake, ${ }^{16-19}$ circulating time and biodistribution. ${ }^{20}$ The protein corona composition and assembly strongly depends on the intrinsic properties of nanoparticles, including the size, shape and surface charges. ${ }^{21-23}$ Additionally, the characteristics of the surrounding environment can also modulate the protein corona formation, such as the 
$\mathrm{pH}^{20}$ or shear stress and flow. ${ }^{24,25}$ Several techniques have been used so far to analyze potein corona, ${ }^{26,27}$ being mass spectrometry-based proteomics ${ }^{28,29}$ and gel electrophoresis ${ }^{30}$ the most extended approaches. Using these techniques, the protein corona analysis is performed in bulk. Recently, super resolution microscopy and, in particular, STORM, is emerging as a powerful tool to characterize the properties of nanomaterials, ${ }^{31}$ including the dynamics of protein corona formation around nanoparticles at the single particle level. ${ }^{32,33}$ Furthermore, STORM is the only current approach that allows visualizing the number and position of individual proteins allowing for a precise spatial mapping. This is particularly relevant in the case of enzyme powered micro- and nanomotors, since their enzymatic distribution is intrinsically heterogeneous, and a certain degree of heterogeneicity in the formation of protein corona around them may also be expected. ${ }^{34}$

Herein, we aim at studying the formation of a protein corona around active urease-powered nanomotors using STORM with two main objectives: i) to study how nanomotor activity affects the formation of protein corona and ii) to study how protein corona formation affects the self-propulsion of nanomotors.

To this end, we synthesized mesoporous silica nanoparticles by using a modified Stöber method, as previously reported, ${ }^{7}$ where cetyltrimethylammonium bromide (CTAB) was employed to generate the mesopores, and triethanolamine (TEOA) and fluorescein isothiocyanate conjugated to (3-aminopropyl)triethoxysilane (FITCAPTES) were used as precursors (6:1 volume). The resulting particles were characterized using Scanning Electron Microscopy (Figure $1 A$ ) which showed monodisperse particles with a mean diameter of $434 \pm 2 \mathrm{~nm}$ (mean \pm s.e.m, $\mathrm{N}=\mathbf{2 0 0}$ ). The inner cylindrical porous structure was verified with transmission electron microscopy (TEM, Figure 1B) where an average pore size of $3 \mathrm{~nm}$ was determined. To functionalize the particles with urease, their surface was first modified with amynopropyltriethoxysilane (APTES) to provide them with available $-\mathrm{NH}_{2}$ functional groups. Urease was subsequently conjugated using glutaraldehyde (GA) as a linker (Figure $1 \mathrm{C}$ ). Nanomotors selfpropel thanks to the conversion of urea into ammonia and carbon dioxide $\left(\left(\mathrm{NH}_{2}\right)_{2} \mathrm{CO}+\mathrm{H}_{2} \mathrm{O} \rightarrow \mathrm{CO}_{2}+2 \mathrm{NH}_{3}\right)$ by urease (Figure $1 \mathrm{D}$ ). ${ }^{34,35}$ To characterize the different functionalization steps, the electrophoretic mobility of the particles was monitored. Dynamic light scattering (DLS) showed the initial unmodified particles to have a surface $\zeta$-potential of -29.5 $\pm 0.34 \mathrm{mV}$ (Smoluchowski), as expected from the presence of silanol groups (Figure $1 \mathrm{E}$ ). After their modification with GA and urease, the $\zeta$-potential of the particles was modified. As expected from the isoelectric points of the surface groups ${ }^{36,37}$, amine modified silica yielded positive surface $\zeta$-potentials and enzyme conjugation recovered the negative surface $\zeta$-potential, confirming the presence of urease on the surface. Urease activity was determined by using a commercial enzyme activity assay kit (Figure $1 \mathrm{~F}$ ).
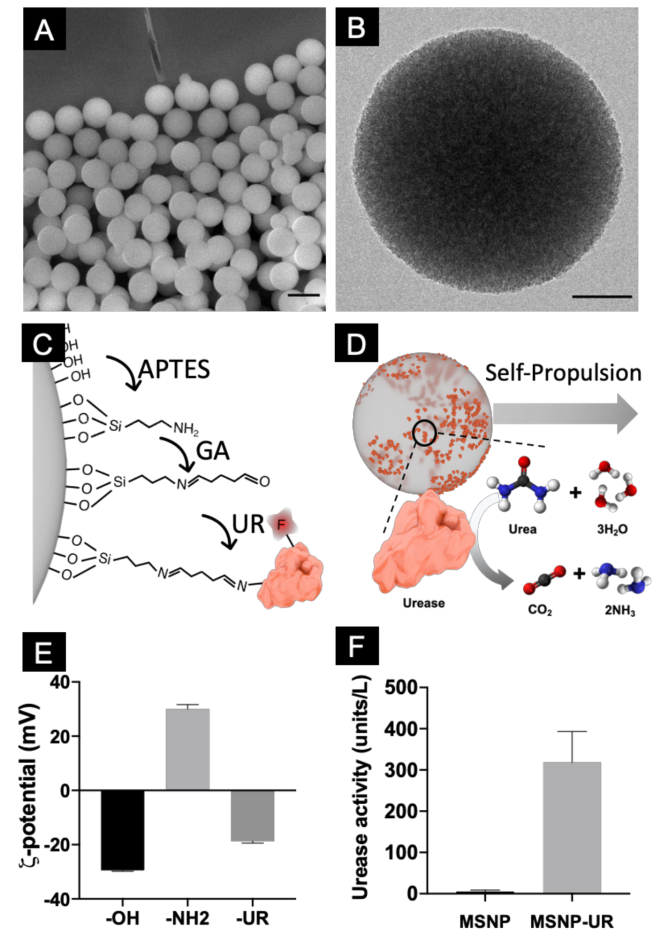

Figure 1. Fabrication and characterization of urease-powered nanomotors. A) SEM micrograph of mesoporous silica nanoparticles. Scale bar $=500 \mathrm{~nm}$. B) Representative TEM micrograph of a mesoporous silica nanoparticle. Scale bar= 10o nm. C) Schematic representation of the functionalization approach. D) Schematic representation of the self-propulsion upon enzyme catalysis. E) $\zeta$ - 
potential characterization of the particles along the functionalization process. F) Enzyme activity of the particles before and after their functionalization with urease.

To generate self-propulsion, an asymmetric distribution of the catalyst is always necessary in order to avoid a net compensation of forces. Recent works have reported that stochastic binding of enzymes onto micron-sized particles results in a non-homogeneous, patchy-like distribution leads to the self-propulsion of biocatalytic micromotors without the need for physical asymmetries like the case of Janus particles. ${ }^{34,3^{8}}$ While this parameter has been thoroughly studied for micron-sized particles, which display a ballistic type of motion, little is known about nano-sized motors, which display a different type of motion known as enhanced diffusion. ${ }^{5}$ For this type of motion dynamics, several asymmetric ${ }^{2,39,40}$ and non-asymmetric structures have been reported..$^{6,41,42}$

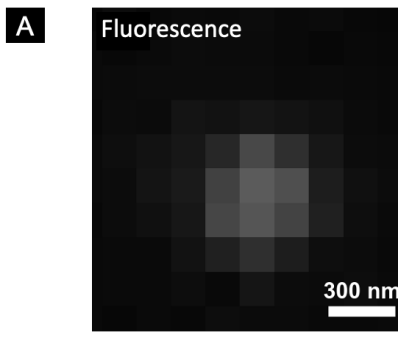

B

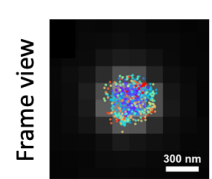

C
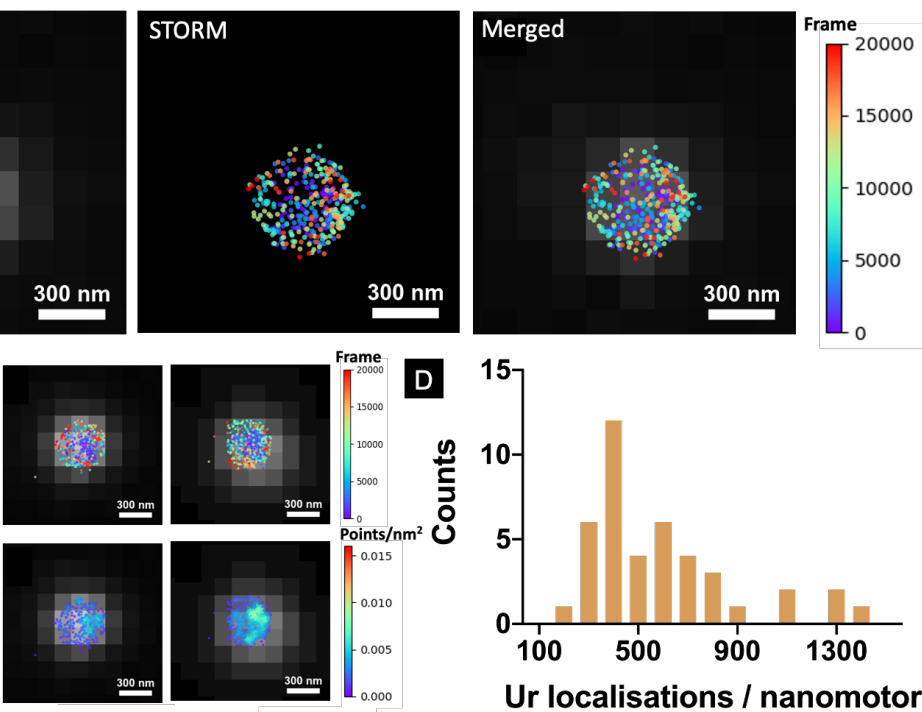

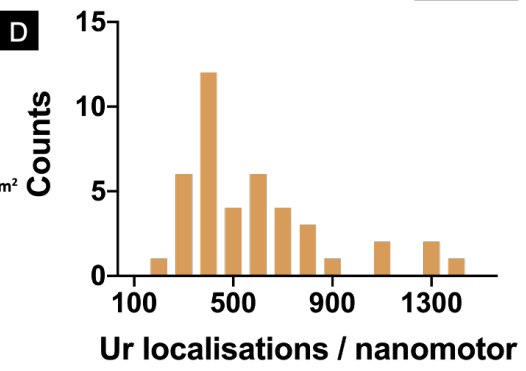

Figure 2. STORM characterization of urease molecules around the nanomotors. A) STORM recorded localisations of Cy5-labelled enzymes are superimposed to the FITC fluorescence image for better visualization. The colour of a localisations represents the frame in which it was recorded. Scale bars are $300 \mathrm{~nm} . \mathrm{B}$ ) Representative STORM images showing urease localizations in a nanomotor. C) Correseponding images showing the localization density, where the distribution of urease can be visualized. D) Histogram of the number of urease localisations per analysed nanomotor. Mean \pm SD $(n=41)$.

However, a robust and systematic analysis of the enzyme distribution onto nano-sized motors is missing. This is particularly relevant since two factors have already been demonstrated to be crucial for self-propulsion of urease micromotors: an asymmetric distribution and the number of enzymes on the motor surface. ${ }^{34}$ Here, we used STORM to detect urease molecules bound to nanomotors surface. For this, urease was previously labelled with Cy5 fluorophore and a calibration to estimate the number of localisations recorded per single labelled urease was performed (Figure $\mathrm{S} 1$ ) ${ }^{34}$ Nanomotors functionalized with $25 \%$ labelled urease were placed onto a glass slide and allowed to precipitate until immobilized. Unbound nanomotors were washed out by replacing the remaining dispersion in PBS solution by the STORM buffer. Figure $2 \mathrm{~A}$ (right) shows the result of merging both conventional fluorescence and STORM images. Figure $2 \mathrm{~B}, 2 \mathrm{C}$ and $\mathrm{S} 2$, show representative examples of urease localizations and enzyme density, respectively. Generally, a non-uniform urease coating of nanomotors was observed (Figures $2 \mathrm{~B}$ and $2 \mathrm{C}$ ), similarly to what has been previously observed for micron- sized urease motors ${ }^{34}$. Using STORM, we were able to determine the amount of urease molecules bound to the nanomotor surface by using a custom-made python-based code, analyzing a minimum amount of 40 nanoparticles per case. First, particle size was determined by the information provided by fluorescence images, being the mean nanoparticle radii $254 \pm 10 \mathrm{~nm}$ (mean \pm s.e.m, $\mathrm{N}=50$ ) being these results consistent with both TEM/SEM analysis. STORM images enabled the quantification of urease molecules bound to the nanoparticle surface, with a mean of $584 \pm 91$ (mean \pm s.e.m, $\mathrm{N}=40$ ).

Motion dynamics was studied by recording the nanomotors either with or without urea, at $100 \mathrm{mM}$ in phosphate buffered saline solution (PBS 1x) which is the optimal concentration of urea at which nanomotors move. ${ }^{6,9,41}$ Bright field videos were recorded for $30 \mathrm{~s}$ at a 25 FPS rate. Nanomotors trajectories were tracked using a custommade python-based code. Figure $3 \mathrm{~A}$ and ${ }_{3} \mathrm{~B}$ show the trajectories of the nanomotors with and without urea, respectively. From the trajectories, we extracted the Mean Squared Displacement (MSD), represented in Figure ${ }_{3} C$. Both with and without fuel, a linear MSD was observed, which denotes a diffusive type of motion dynamics. 
However, in the case of nanomotors in $100 \mathrm{mM}$ urea, the slope of the MSD was significantly higher, indicating enhanced diffusion (Figure $3 \mathrm{C}$ ). $., 43,44$

Figure $3 \mathrm{D}$ shows the distribution of obtained diffusion coefficients, being them significantly higher $(P=0.0035)$ in the case of $100 \mathrm{mM}$ urea.
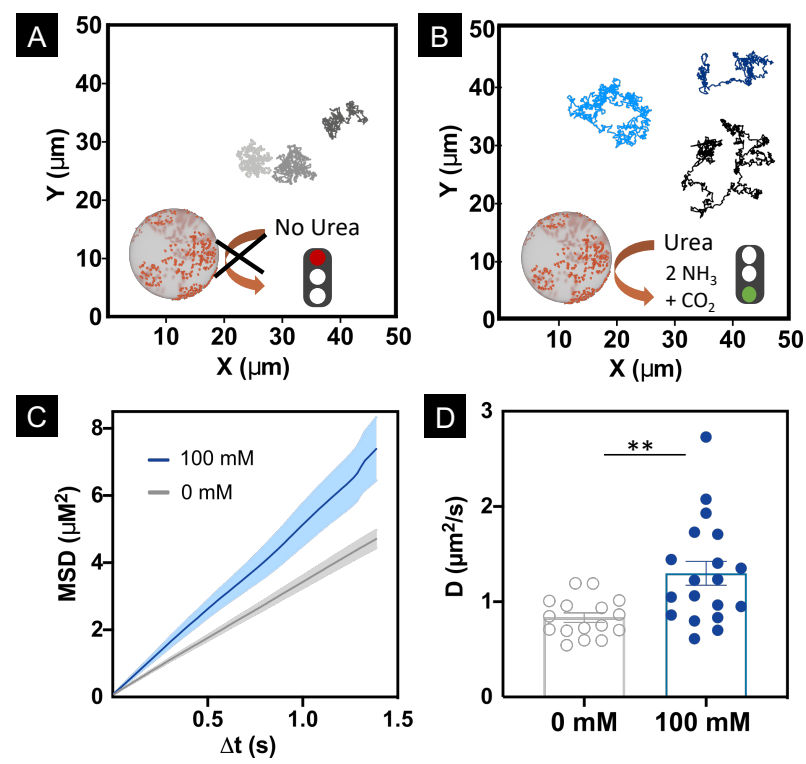

Figure 3. Motion dynamics of urease nanomotors. A) Trajectories of nanomotors in the absence of fuel. B) Trajectories of nanomotors in the presence of $100 \mathrm{mM}$ urea. C) Comparison between the MSD in the presence or absence of fuel. Results are shown as the mean \pm s.e.m $(n=20)$ D) Diffusion coefficient of the nanomotors calculated from the MSD. Results are shown as the mean \pm s.e.m $(n=20)$.

Addressing the performance of biomedical micro- and nanomotors in complex biological fluids is a critical aspect, since certain physiological conditions may hamper their motility. In this study, we monitored the motility of particles using PBS, which contains different salts and ionic species at a physiologically relevant concentration. The presence of ionic species has been recently demonstrated to impede the motion of micron-sized motors powered by urease. ${ }^{45}$ However, in the case of nano-sized motors, their motion persist in different types of media, including PBS, ${ }^{6,46}$ urine $^{41}$ and blood ${ }^{10}$, probably due to their different type of motion mechanism. Nonetheless, biological fluids not only contain salts and ionic species and other components such as proteins might interact with the nanomotor surface. Here, to investigate the effect of the activity of nanomotors on the formation of protein corona in a quantitative manner, we incubated urease-nanomotors with Cy5-labelled serum proteins, either in the presence or absence of $100 \mathrm{mM}$ urea (Figure $4 \mathrm{~A}$ ). For this, urease nanomotors were incubated for 30 min in a solution containing non-labelled Fetal Bovine Serum (FBS), 5\% Cy5 labelled FBS and o or $100 \mathrm{mM}$ urea for the control and active samples, respectively (Figure A). As in the previous experiments, green fluorescence was used to localize the nanomotor boundary and the images were superimposed with the STORM images (Figure $4 \mathrm{~B}$ and ${ }_{4} \mathrm{C}$ ), where labelled serum proteins were quantified. Figure $4 \mathrm{D}$ shows a histogram depicting the distribution of FBS detections per nanomotor. In the presence of $100 \mathrm{mM}$ urea, the peak of the histogram is significantly shifted to the left, indicating a lower average FBS detection per nanomotors, compared to the nanomotors without fuel. Figure $4 \mathrm{E}$ shows a comparison of the average detected FBS points per nanomotor. Surprisingly, around $20 \%$ reduction on the FBS localizations was found between control $(615 \pm 24$ localizations, mean \pm s.e.m.) and active nanomotors ( $486 \pm 21$ localizations, mean \pm s.e.m.). These results indicate a significant reduction $(P=0.002)$ of protein corona formation around nanomotors when they are active. This effect could be explained by two factors. First, urease activity of micromotors has been already reported to modify the local surrounding environment, leading to a $\mathrm{pH}$ increase. ${ }^{8,47}$ The formation and stability of protein corona has shown sensitivity to different $\mathrm{pH}^{48-50}$ and particle surface charges. ${ }^{51}$ The changes in $\mathrm{pH}$ induced by urease activity could thus result in an alteration of the physicochemical properties of the particle surface and the serum proteins, ultimately affecting the interactions between both. Second, nanomotors chemical activity often leads to the creation of a shear flow around them. ${ }^{44}$ This is also a very interesting feature, since protein corona has shown to be dependent not 
only on the physicochemical properties of the particles but also on the presence of shear stress and shear flows. $^{24,25,52}$

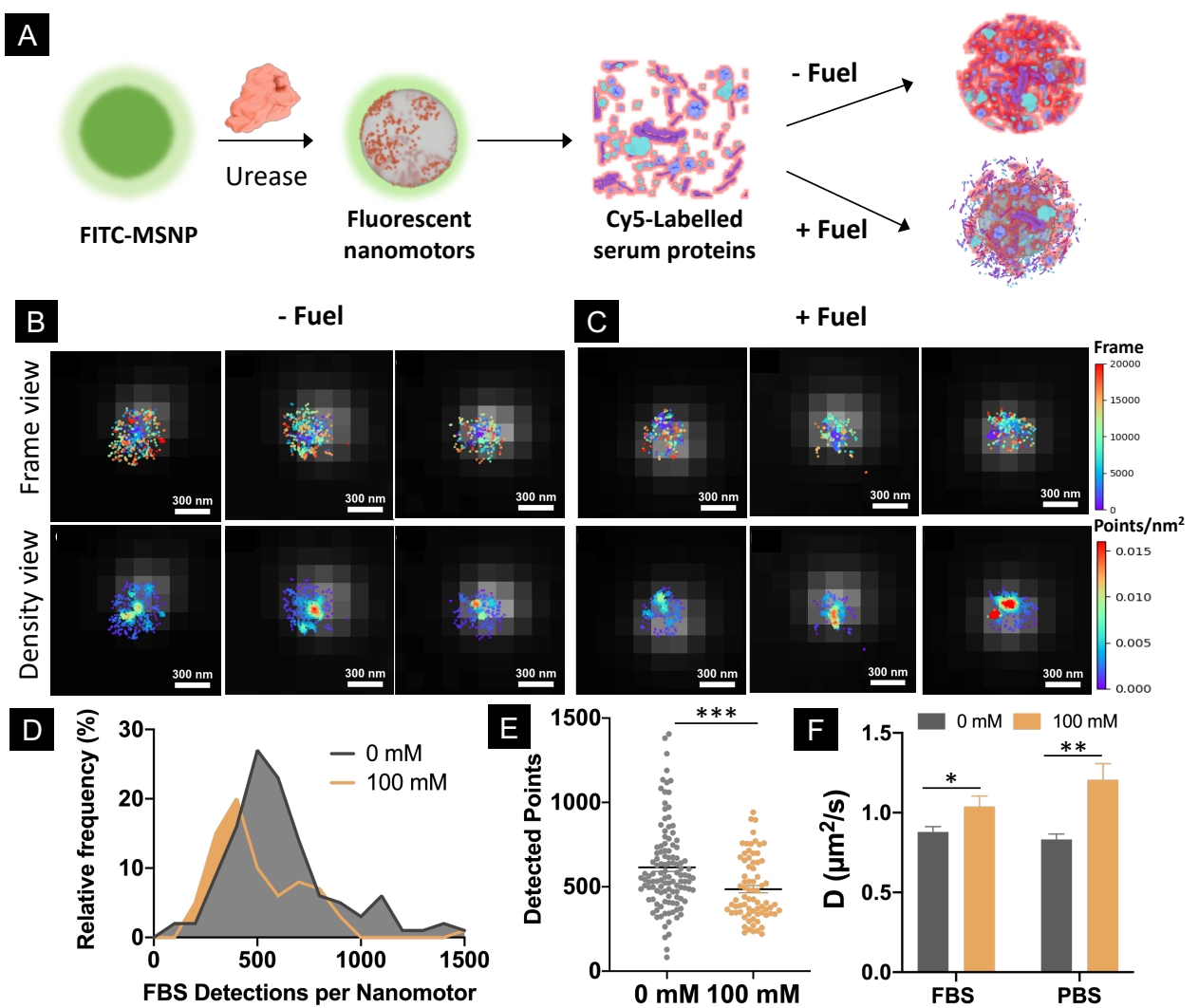

Figure 4. Protein corona formation around active nanomotors. A) Schematic representation of the experimental approach, where fluorescent nanoparticles (FITC-MSNP) were functionalized with non-labelled urease and incubated with Cy5-labelled serum proteins either in the presence or absence of fuel. B) Representative STORM images of nanomotors after their incubation with labelled FBS in the absence of fuel, showing FBS localizations (top) and FBS density (bottom). C) Representative STORM images of nanomotors after their incubation with labelled FBS in the presence of fuel, showing FBS localizations (top) and FBS density (bottom). D) Histogram of the FBS detections per nanomotor distribution. E) Comparison of the average detected FBS proteins in nanomotors with and without urea. F) Comparison of the diffusion coefficients of the nanomotors in different conditions. Results are shown as the mean \pm s.e.m.

Since the self-propulsion of nanomotors is generated by the decomposition of urea catalyzed by urease, the presence of a protein corona on the particle surface could hamper the substrate and product exchanges, limiting the motion performance. For this reason, we analyzed the motion of nanomotors after the formation of protein corona. For this, we incubated the nanomotors with FBS as described previously, following the same procedure as for the STORM imaging experiments. Then, the motors were resuspended in PBS $1 \mathrm{x}$ and their motion was analysed as described above. Results showed a significant increase $(P=0.047)$ in the diffusion coefficient of the nanomotors in $100 \mathrm{mM}$ urea, constituting an increase of $18 \%$ respect to the control (o $\mathrm{mM}$ urea). This increase was slightly lower than the one observed for the nanomotors swimming in PBS, without being previously incubated with FBS, where a $44 \%$ increase in the diffusion coefficient was found. These results indicate that urease powered nanomotors are able to swim regardless of the diminished protein corona formation around their surface. However, their performance is slightly limited compared to the bare nanomotors, indicating that future strategies to further reduce protein corona might be desired. 

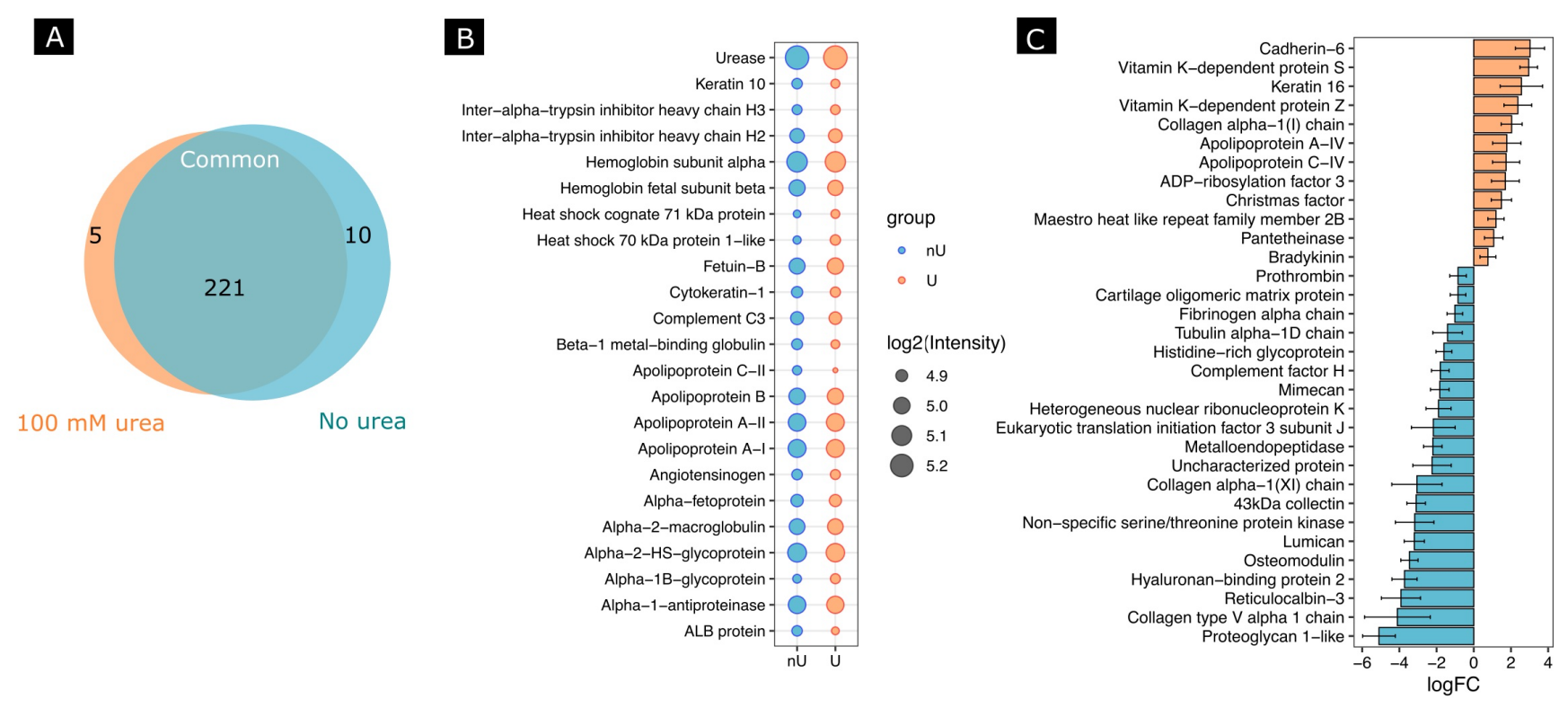

Figure 5. Proteomic analysis of protein corona composition around nanomotors when incubated 30 min with FBS. A) Venn diagram representing the number of proteins only present in the nanomotors exposed to urea, no urea and the number of proteins present in both conditions. B) Dotplot displaying the most abundant proteins, according to their intensity. In each case, results are shown as the mean $\log _{2}$ (Intensity) of three independent biological replicates. nU: no urea; U: 100 mM urea. C) Fold-change of the most significant proteins for the $100 \mathrm{mM}$ urea conditions (light orange) with respect to no urea (light blue) condition. Results are shown as the mean \pm SD.

Next, we investigated whether the activity of the nanomotors resulted in a change in protein corona composition. For this, after incubating nanomotors with FBS for 30 min with or without fuel, we performed a proteomic analysis by digesting adsorbed proteins using a trypsin treatment, followed by protein identification using HPLCMass Spectrometry analysis. We identified a total amount of 321 proteins, from which 236 protein groups were quantified. A differential expression analysis between $100 \mathrm{mM}$ urea and no urea conditions was performed. Figure 5 A shows a venn diagram of the proteins found for each condition and in common for both conditions. When we selected the 20 most abundant proteins for each condition (Figure $5 \mathrm{~B}$ ), the changes in aboundance were nonsignificant, although the tendency was to find a lower aboundance for most proteins in the case of urea condition. Interestingly, for the non-urea condition, the heat shock cognate $71 \mathrm{kDa}$ protein and Heat shock $70 \mathrm{kDa}$ protein 1-like were among the most abundant, which was not the case for urea condition. Other examples are Apolipoprotien C-II and ALB protein, which were among the 20 most abundant proteins in urea conditions but not in non-urea conditions.

Moreover, several significant proteins were identified using standard cutoffs for fold change $(|\mathrm{FC}|>1.5)$ and adjusted p-value (padj < 0.05) when comparing urea and non-urea conditions (Figure $S_{3}$ ). In this regard, 13 proteins were shown to be up-regulated and 21 down-regulated (Figure ${ }_{5} \mathrm{C}$ ).

This study reports for the first time the relationship behind enzyme-driven motion and protein corona, highlighing the relevance of extending these studies to the micro- and nanomotors community, since the effects on their propulsion capabilities might depend on the particle properties and their interaction with biological media. We made use of superresolution microscopy to unveil the protein corona formation on nanomotors in the presence and absence of chemical fuel, and we ideantified the type and quantified its variation by proteomics analysis. Moreover, our observations show that, active nanomotors can reduce the protein corona formation, which might be desirable for certain biomedical applications. Noteworthy, the changes in protein corona are not only qualitative but also quantitative, where the impact in different biological interactions such as cell-nanoparticle interactions will need to be considered in the near future. 


\section{Supporting Information.}

Supporting Information: Materials and methods, characterization of nanomotors enzymatic activity, characterization of Cy5urease labelling by STORM. (PDF) (file type, i.e., PDF)

brief description (file type, i.e., PDF)

\section{AUTHOR INFORMATION}

\section{* Corresponding Author}

Tania Patiño; Chemistry Department, University of Rome, Tor Vergata, Via della Ricerca Scientifica, oo133 Rome, Italy; Email: tania.patino@uniromaz.eu

Institute for Bioengineering of Catalonia (IBEC), The Barcelona Institute of Science and Technology (BIST), Baldiri i Reixac 10-12, o8028 Barcelona, Spain tpatino@ibecbarcelona.eu

Lorenzo Albertazzi - Department of Biomedical Engineering, Institute for Complex Molecular Systems (ICMS), Eindhoven University of Technology, $5612 A Z$ Eindhoven, The Netherlands

Institute for Bioengineering of Catalonia (IBEC), The Barcelona Institute of Science and Technology (BIST), Baldiri i Reixac 10-12, o8028 Barcelona, Spain. E-mail: lalbertazzi@ibecbarcelona.eu

Samuel Sánchez - Institute for Bioengineering of Catalonia (IBEC), The Barcelona Institute of Science and Technology

(BIST), Baldiri i Reixac 10-12, o8028 Barcelona, Spain; ICREA, Pg. Lluís Companys 23, Barcelona, o8010, Spain; E-

mail: ssanchez@ibecbarcelona.eu

Present Addresses

†ETH Zürich, Zurich, 8092 Switzerland

\section{Author Contributions}

$\$$ These authors contributed equally.

Funding Sources

No competing financial interests have been declared.

\section{ACKNOWLEDGMENT}

The research leading to these results has received funding from the Spanish MINECO (BOTSinFluids project), the Foundation BBVA (MEDIROBOTS project), the CERCA program by the Generalitat de Catalunya and the CaixaImpulse program by La Caixa Foundation (TERANOBOTS project). This project has received funding from the European Research Council (ERC) under the European Union's Horizon 2020 research and innovation programme (grant agreement No 866348; iNanoSwarms). T.P. thanks the European Union's Horizon 2020 research and innovation program, under the Marie Skłodowska-Curie Individual Fellowship (H2020-MSCA-IF2018, DNA-bots). S.P. and L.A acknowledge the financial support by the Spanish Ministry of Science and Innovation (PID2019-10945oRB-Ioo/AEI /10.13039/501100011033), European Research Council/Horizon 2020 (ERC-StG-757397), "la Caixa" Foundation (ID 100010434), and by the Generalitat de Catalunya (through the CERCA program and 2017 SGR 01536). Mass spectrometry/Proteomics was performed at the IRB Barcelona Mass Spectrometry and Proteomics Core Facility, which is a member of ProteoRed, PRB3-ISCIII, supported by grant PRB3 (IPT17/oo19 - ISCIII-SGEFI / ERDF) and is granted in the framework of the 2014-2020 ERDF Operational Programme in Catalonia, co-financed by the European Regional Development Fund (ERDF, Reference: IU16-015983).

\section{REFERENCES}

(1) Sánchez, S.; Soler, L.; Katuri, J. Chemically Powered Micro- and Nanomotors. Angew. Chemie Int. Ed. 2015, 54 (5), $1414-1444$. https://doi.org/10.1002/anie.201406096.

(2) Schattling, P. S.; Ramos-Docampo, M. A.; Salgueiriño, V.; Städler, B. Double-Fueled Janus Swimmers with Magnetotactic Behaviour. ACS Nano 2017, 11 (4), 3973-3983. https://doi.org/10.1021/acsnano.7boo441.

(3) Abdelmohsen, L. K. E. A.; Nijemeisland, M.; Pawar, G. M.; Janssen, G.-J. A.; Nolte, R. J. M.; van Hest, J. C. M.; Wilson, D. A.; Nanomotor, E. S.; Abdelmohsen, L. K. E. A.; Nijemeisland, M.; Pawar, G. M.; Janssen, G.-J. A.; Nolte, R. J. M.; Hest, J. C. M. Van; Wilson, D. A. Dynamic Loading and Unloading of Proteins in Polymeric Stomatocytes: Formation of an Enzyme-Loaded Supramolecular Nanomotor. ACS Nano 2016, 10 (2), 2652-266o. https://doi.org/10.1021/acsnano.5bo7689.

(4) Ma, X.; Hortelao, A. C.; Patiño, T.; Sanchez, S. Enzyme Catalysis to Power Micro / Nano Machines. ACS Na 2016, 10 (10), $9111-9122$. https://doi.org/10.1021/acsnano.6bo4108.

(5) Patiño, T.; Arqué, X.; Mestre, R.; Palacios, L.; Sánchez, S. Fundamental Aspects of Enzyme-Powered Micro- and Nanoswimmers. Acc. Chem. Res. 2018, 51 (11), 2662-2671. https://doi.org/10.1021/acs.accounts.8boo288. 
(6) Hortelão, A. C.; Patiño, T.; Perez-Jiménez, A.; Blanco, À.; Sánchez, S. Enzyme-Powered Nanobots Enhance Anticancer Drug Delivery. Adv. Funct. Mater. 2017, 28 (25), 1705086. https://doi.org/10.1002/adfm.201705086.

(7) Hortelão, A. C.; Carrascosa, R.; Murillo-Cremaes, N.; Patiño, T.; Sánchez, S. Targeting 3D Bladder Cancer Spheroids with UreasePowered Nanomotors. ACS Nano 2019, 13 (1), 429-439. https://doi.org/10.1021/acsnano.8bo6610.

(8) Patino, T.; Porchetta, A.; Jannasch, A.; Lladó, A.; Stumpp, T.; Schäffer, E.; Ricci, F.; Sánchez, S. Self-Sensing Enzyme-Powered Micromotors Equipped with PH-Responsive DNA Nanoswitches. Nano Lett. 2019, 19 (6), 3440-3447.

https://doi.org/10.1021/acs.nanolett.8bo4794.

(9) Arqué, X.; Romero-Rivera, A.; Feixas, F.; Patiño, T.; Osuna, S.; Sánchez, S. Intrinsic Enzymatic Properties Modulate the Self-Propulsion of Micromotors. Nat. Commun. 2019, 10 (1), 2826. https://doi.org/10.1038/s41467-019-10726-8.

(10) Tang, S.; Zhang, F.; Gong, H.; Wei, F.; Zhuang, J.; Karshalev, E.; Esteban-Fernández de Ávila, B.; Huang, C.; Zhou, Z.; Li, Z.; Yin, L.; Dong, H.; Fang, R. H.; Zhang, X.; Zhang, L.; Wang, J. Enzyme-Powered Janus Platelet Cell Robots for Active and Targeted Drug Delivery. Sci. Robot. 2020, 5 (43), eaba6137. https://doi.org/10.1126/scirobotics.aba6137.

(11) Wang, W.; Zhou, C. A Journey of Nanomotors for Targeted Cancer Therapy: Principles, Challenges, and a Critical Review of the Stateof-the-Art. Adv. Healthc. Mater. 2020, 2001236. https://doi.org/10.1002/adhm.202001236.

(12) Monopoli, M. P.; Åberg, C.; Salvati, A.; Dawson, K. A. Biomolecular Coronas Provide the Biological Identity of Nanosized Materials. Nat. Nanotechnol. 2012, 7 (12), 779-786. https://doi.org/10.1038/nnano.2012.207.

(13) Dawson, K. A.; Yan, Y. Current Understanding of Biological Identity at the Nanoscale and Future Prospects. Nat. Nanotechnol. 2021. https://doi.org/10.1038/s41565-021-0086o-o.

(14) Mohammad-Beigi, H.; Hayashi, Y.; Zeuthen, C. M.; Eskandari, H.; Scavenius, C.; Juul-Madsen, K.; Vorup-Jensen, T.; Enghild, J. J.; Sutherland, D. S. Mapping and Identification of Soft Corona Proteins at Nanoparticles and Their Impact on Cellular Association. Nat. Commun. 2020, 11 (1), 4535. https://doi.org/10.1038/s41467-020-18237-7.

(15) Salvati, A.; Pitek, A. S.; Monopoli, M. P.; Prapainop, K.; Bombelli, F. B.; Hristov, D. R.; Kelly, P. M.; Åberg, C.; Mahon, E.; Dawson, K. A. Transferrin-Functionalized Nanoparticles Lose Their Targeting Capabilities When a Biomolecule Corona Adsorbs on the Surface. Nat. Nanotechnol. 2013, 8 (2), 137-143. https://doi.org/10.1038/nnano.2012.237.

(16) Wang, F.; Yu, L.; Monopoli, M. P.; Sandin, P.; Mahon, E.; Salvati, A.; Dawson, K. a. The Biomolecular Corona Is Retained during Nanoparticle Uptake and Protects the Cells from the Damage Induced by Cationic Nanoparticles until Degraded in the Lysosomes. Nanomedicine 2013, 9 (8), 1159-1168. https://doi.org/10.1016/j.nano.2013.04.010.

(17) Yan, Y.; Gause, K. T.; Kamphuis, M. M. J.; Ang, C.-S.; O’Brien-Simpson, N. M.; Lenzo, J. C.; Reynolds, E. C.; Nice, E. C.; Caruso, F. Differential Roles of the Protein Corona in the Cellular Uptake of Nanoporous Polymer Particles by Monocyte and Macrophage Cell Lines. ACS Nano 2013, 7 (12), 10960-10970. https://doi.org/10.1021/nn404481f.

(18) Ritz, S.; Schöttler, S.; Kotman, N.; Baier, G.; Musyanovych, A.; Kuharev, J.; Landfester, K.; Schild, H.; Jahn, O.; Tenzer, S.; Mailänder, V. Protein Corona of Nanoparticles: Distinct Proteins Regulate the Cellular Uptake. Biomacromolecules 2015. https://doi.org/10.1021/acs.biomac.5boo108.

(19) Lesniak, A.; Fenaroli, F.; Monopoli, M. P.; Åberg, C.; Dawson, K. A.; Salvati, A. Effects of the Presence or Absence of a Protein Corona on Silica Nanoparticle Uptake and Impact on Cells. ACS Nano 2o12, 6 (7), 5845-5857. https://doi.org/10.1021/nn300223w.

(20) Tekie, F. S. M.; Hajiramezanali, M.; Geramifar, P.; Raoufi, M.; Dinarvand, R.; Soleimani, M.; Atyabi, F. Controlling Evolution of Protein Corona: A Prosperous Approach to Improve Chitosan-Based Nanoparticle Biodistribution and Half-Life. Sci. Rep. 2020,10 (1), 9664. https://doi.org/10.1038/s41598-020-66572-y.

(21) Walkey, C. D.; Olsen, J. B.; Song, F.; Liu, R.; Guo, H.; Olsen, D. W. H.; Cohen, Y.; Emili, A.; Chan, W. C. W. Protein Corona Fingerprinting Predicts the Cellular Interaction of Gold and Silver Nanoparticles. ACS Nano 2014, 8 (3), 2439-2455. https://doi.org/10.1021/nn406o18q.

(22) Tenzer, S.; Docter, D.; Kuharev, J.; Musyanovych, A.; Fetz, V.; Hecht, R.; Schlenk, F.; Fischer, D.; Kiouptsi, K.; Reinhardt, C.; Landfester, K.; Schild, H.; Maskos, M.; Knauer, S. K.; Stauber, R. H. Rapid Formation of Plasma Protein Corona Critically Affects Nanoparticle Pathophysiology. Nat Nano 2013, 8 (10), 772-781.

(23) García-Álvarez, R.; Hadjidemetriou, M.; Sánchez-Iglesias, A.; Liz-Marzán, L. M.; Kostarelos, K. In Vivo Formation of Protein Corona on Gold Nanoparticles. The Effect of Their Size and Shape. Nanoscale 2o18, 10 (3), 1256-1264. https://doi.org/10.1039/C7NRo8322J.

(24) Palchetti, S.; Pozzi, D.; Capriotti, A. L.; Barbera, G. La; Chiozzi, R. Z.; Digiacomo, L.; Peruzzi, G.; Caracciolo, G.; Laganà, A. Influence of Dynamic Flow Environment on Nanoparticle-Protein Corona: From Protein Patterns to Uptake in Cancer Cells. Colloids Surfaces B Biointerfaces 2017, 153, 263-271. https://doi.org/10.1016/j.colsurfb.2017.02.037.

(25) Jayaram, D. T.; Pustulka, S. M.; Mannino, R. G.; Lam, W. A.; Payne, C. K. Protein Corona in Response to Flow: Effect on Protein Concentration and Structure. Biophys. J. 2018, 115 (2), 209-216. https://doi.org/10.1016/j.bpj.2018.02.036.

(26) Carrillo-Carrion, C.; Carril, M.; Parak, W. J. Techniques for the Experimental Investigation of the Protein Corona. Curr. Opin. Biotechnol. 2017, 46, 106-113. https://doi.org/10.1016/j.copbio.2017.02.009.

(27) Capriotti, A. L.; Caracciolo, G.; Cavaliere, C.; Colapicchioni, V.; Piovesana, S.; Pozzi, D.; Laganà, A. Analytical Methods for Characterizing the Nanoparticle-Protein Corona. Chromatographia 2014, 77 (11-12), 755-769. https://doi.org/10.1007/s10337-014-2677-x. Blume, J. E.; Manning, W. C.; Troiano, G.; Hornburg, D.; Figa, M.; Hesterberg, L.; Platt, T. L.; Zhao, X.; Cuaresma, R. A.; Everley, P. A.; Ko, M.; Liou, H.; Mahoney, M.; Ferdosi, S.; Elgierari, E. M.; Stolarczyk, C.; Tangeysh, B.; Xia, H.; Benz, R.; Siddiqui, A.; Carr, S. A.; Ma, P.; Langer, R.; Farias, V.; Farokhzad, O. C. Rapid, Deep and Precise Profiling of the Plasma Proteome with Multi-Nanoparticle Protein Corona. Nat. Commun. 2020, 11 (1), 3662. https://doi.org/10.1038/s41467-020-17033-7.

(29) Papafilippou, L.; Claxton, A.; Dark, P.; Kostarelos, K.; Hadjidemetriou, M. Protein Corona Fingerprinting to Differentiate Sepsis from Non-Infectious Systemic Inflammation. Nanoscale 2020, 12 (18), 10240-10253. https://doi.org/10.1039/DoNRo2788J.

(30) Monopoli, M. P.; Walczyk, D.; Campbell, A.; Elia, G.; Lynch, I.; Baldelli Bombelli, F.; Dawson, K. A. Physical-Chemical Aspects of Protein Corona: Relevance to in Vitro and in Vivo Biological Impacts of Nanoparticles. J. Am. Chem. Soc. 2011, 133 (8), $2525-2534$. https://doi.org/10.1021/ja107583h.

(31) Pujals, S.; Feiner-Gracia, N.; Delcanale, P.; Voets, I.; Albertazzi, L. Super-Resolution Microscopy as a Powerful Tool to Study Complex Synthetic Materials. Nat. Rev. Chem. 2019, 3 (2), 68-84. https://doi.org/10.1038/s41570-018-0070-2.

(32) Feiner-Gracia, N.; Beck, M.; Pujals, S.; Tosi, S.; Mandal, T.; Buske, C.; Linden, M.; Albertazzi, L. Super-Resolution Microscopy Unveils Dynamic Heterogeneities in Nanoparticle Protein Corona. Small 2017, 13 (41), 1701631. https://doi.org/10.1002/smll.201701631.

(33) Clemments, A. M.; Botella, P.; Landry, C. C. Spatial Mapping of Protein Adsorption on Mesoporous Silica Nanoparticles by Stochastic Optical Reconstruction Microscopy. J. Am. Chem. Soc. 2017, 139 (11), 3978-3981. https://doi.org/10.1021/jacs.7bo1118.

(34) Patiño, T.; Feiner-Gracia, N.; Arqué, X.; Miguel-López, A.; Jannasch, A.; Stumpp, T.; Schäffer, E.; Albertazzi, L.; Sánchez, S. Influence of Enzyme Quantity and Distribution on the Self-Propulsion of Non-Janus Urease-Powered Micromotors. J. Am. Chem. Soc. 2018, 140 (25), 
De Corato, M.; Arqué, X.; Patiño, T.; Arroyo, M.; Sánchez, S.; Pagonabarraga, I. Self-Propulsion of Active Colloids via Ion Release:

Theory and Experiments. Phys. Rev. Lett. 2020, 124 (10), 1080o1. https://doi.org/10.1103/PhysRevLett.124.1080o1.

(36) Wu, Z.; Xiang, H.; Kim, T.; Chun, M. S.; Lee, K. Surface Properties of Submicrometer Silica Spheres Modified with Aminopropyltriethoxysilane and Phenyltriethoxysilane. J. Colloid Interface Sci. 2006, 304 (1), 119-124. https://doi.org/10.1016/j.jcis.2006.08.055.

(37) Sumner, J. B.; Hand, D. B. The Isoelectric Point of Crystalline Urease. J. Am. Chem. Soc. 1929, 51 (4), $1255^{-1260 .}$ https://doi.org/10.1021/jao1379ao39.

(38) Post, R. A. J.; van der Zwaag, D.; Bet, G.; Wijnands, S. P. W.; Albertazzi, L.; Meijer, E. W.; van der Hofstad, R. W. A Stochastic View on Surface Inhomogeneity of Nanoparticles. Nat. Commun. 2019, 10 (1), 1663. https://doi.org/10.1038/s41467-019-09595-y.

(39) Ma, X.; Sánchez, S. Bio-Catalytic Mesoporous Janus Nano-Motors Powered by Catalase Enzyme. Tetrahedron 2017. https://doi.org/10.1016/j.tet.2017.06.048.

(40) Ma, X.; Jannasch, A.; Albrecht, U.-R.; Hahn, K.; Miguel-López, A.; Schäffer, E.; Sánchez, S. Enzyme-Powered Hollow Mesoporous Janus Nanomotors. Nano Lett. 2015, 15 (10), 7043-7050. https://doi.org/10.1021/acs.nanolett.5bo310o.

(41) Hortelao, A. C.; Carrascosa, R.; Murillo-Cremaes, N.; Patino, T.; Sánchez, S.; Hortelão, A. C.; Carrascosa, R.; Murillo-Cremaes, N.; Patiño, T.; Sánchez, S. Targeting 3D Bladder Cancer Spheroids with Urease-Powered Nanomotors. ACS Nano 2o19, 13 (1), 429-439. https://doi.org/10.1021/acsnano.8bo6610.

(42) Dey, K. K.; Zhao, X.; Tansi, B. M.; Méndez-Ortiz, W. J.; Córdova-Figueroa, U. M.; Golestanian, R.; Sen, A. Micromotors Powered by Enzyme Catalysis. Nano Lett. 2015, 15 (12), 8311-8315. https://doi.org/10.1021/acs.nanolett.5bo3935.

(43) Dunderdale, G.; Ebbens, S.; Fairclough, P.; Howse, J. Importance of Particle Tracking and Calculating the Mean-Squared Displacement in Distinguishing Nanopropulsion from Other Processes. Langmuir 2012, 28, 10997-11006.

(44) Howse, J. R.; Jones, R. A. L.; Ryan, A. J.; Gough, T.; Vafabakhsh, R.; Golestanian, R. Self-Motile Colloidal Particles : From Directed Propulsion to Random Walk. Phys. Rev. Lett. 2007, 99 (4), 8-11. https://doi.org/10.1103/PhysRevLett.99.048102.

(45) Arqué, X.; Andrés, X.; Mestre, R.; Ciraulo, B.; Ortega Arroyo, J.; Quidant, R.; Patiño, T.; Sánchez, S. Ionic Species Affect the SelfPropulsion of Urease-Powered Micromotors. Research 2020, 2020, 1-14. https://doi.org/10.34133/2020/2424972.

(46) Llopis-Lorente, A.; García-Fernández, A.; Murillo-Cremaes, N.; Hortelão, A. C.; Patiño, T.; Villalonga, R.; Sancenón, F.; Martínez-Máñez, R.; Sánchez, S. Enzyme-Powered Gated Mesoporous Silica Nanomotors for On-Command Intracellular Payload Delivery. ACS Nano 2019, 13 (10), 12171-12183. https://doi.org/10.1021/acsnano.9bo6706.

(47) Walker, D.; Ka sdorf, B. T.; Jeong, H. H. H.-H.; Lieleg, O.; Fischer, P.; Käsdorf, B. T.; Jeong, H. H. H.-H.; Lieleg, O.; Fischer, P. Enzymatically Active Biomimetic Micropropellers for the Penetration of Mucin Gels. Sci. Adv. 2015, 1 (11), e1500501-e1500501. https://doi.org/10.1126/sciadv.1500501.

(48) Wang, W.; Huang, Z.; Li, Y.; Wang, W.; Shi, J.; Fu, F.; Huang, Y.; Pan, X.; Wu, C. Impact of Particle Size and PH on Protein Corona Formation of Solid Lipid Nanoparticles: A Proof-of-Concept Study. Acta Pharm. Sin. B 2o2o. https://doi.org/10.1016/j.apsb.2020.10.023.

(49) Raoufi, M.; Hajipour, M. J.; Kamali Shahri, S. M.; Schoen, I.; Linn, U.; Mahmoudi, M. Probing Fibronectin Conformation on a Protein Corona Layer around Nanoparticles. Nanoscale 2018, 10 (3), 1228-1233. https://doi.org/10.1039/C7NRo697oG.

(5o) Gorshkov, V.; Bubis, J. A.; Solovyeva, E. M.; Gorshkov, M. V.; Kjeldsen, F. Protein Corona Formed on Silver Nanoparticles in Blood Plasma Is Highly Selective and Resistant to Physicochemical Changes of the Solution. Environ. Sci. Nano 2019, 6 (4), $1089-1098$. https://doi.org/10.1039/C8ENo1054D.

(51) Lundqvist, M.; Stigler, J.; Elia, G.; Lynch, I.; Cedervall, T.; Dawson, K. A. Nanoparticle Size and Surface Properties Determine the Protein Corona with Possible Implications for Biological Impacts. Proc. Natl. Acad. Sci. 2008, 105 (38), 14265-14270. https://doi.org/10.1073/pnas.0805135105.

(52) Bonvin, D.; Aschauer, U.; Alexander, D. T. L.; Chiappe, D.; Moniatte, M.; Hofmann, H.; Mionić Ebersold, M. Protein Corona: Impact of Lymph Versus Blood in a Complex In Vitro Environment. Small 2017, 13 (29), 1700409. https://doi.org/10.1002/smll.201700409. 


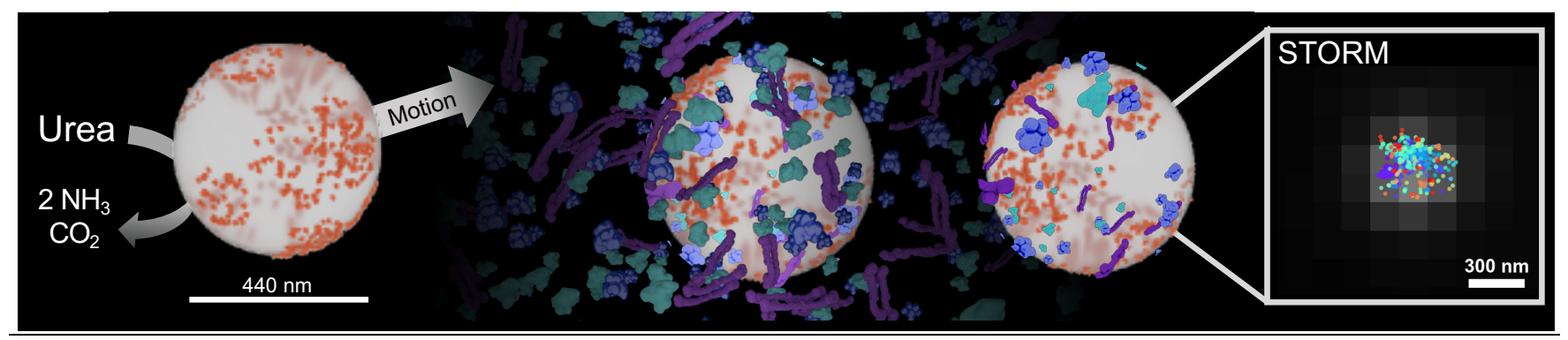

